\title{
Characterization of the Hokuyo URG-04LX Laser Rangefinder for Mobile Robot Obstacle Negotiation
}

\author{
Yoichi Okubo*, Cang Ye**, and Johann Borenstein* \\ * The University of Michigan, 2260 Hayward Street, Ann Arbor MI 48109, USA \\ ** University of Arkansas at Little Rock, 2801 S. University Avenue, ETAS 575 Little Rock, AR 72204 \\ Email addresses in author order: yokb@umich.edu, cxye@ualr.edu, johannb@umich.edu
}

\begin{abstract}
This paper presents a characterization study of the Hokuyo URG-04LX scanning laser rangefinder (LRF). The Hokuyo LRF is similar in function to the Sick LRF, which has been the de-facto standard range sensor for mobile robot obstacle avoidance and mapping applications for the last decade. Problems with the Sick LRF are its relatively large size, weight, and power consumption, allowing its use only on relatively large mobile robots. The Hokuyo LRF is substantially smaller, lighter, and consumes less power, and is therefore more suitable for small mobile robots. The question is whether it performs just as well as the Sick LRF in typical mobile robot applications.

In 2002, two of the authors of the present paper published a characterization study of the Sick LRF. For the present paper we used the exact same test apparatus and test procedures as we did in the 2002 paper, but this time to characterize the Hokuyo LRF. As a result, we are in the unique position of being able to provide not only a detailed characterization study of the Hokuyo LRF, but also to compare the Hokuyo LRF with the Sick LRF under identical test conditions. Among the tested characteristics are sensitivity to a variety of target surface properties and incidence angles, which may potentially affect the sensing performance. We also discuss the performance of the Hokuyo LRF with regard to the mixed pixels problem associated with LRFs. Lastly, the present paper provides a calibration model for improving the accuracy of the Hokuyo LRF.
\end{abstract}

\section{INTRODUCTION}

Range sensing is a crucial element of any obstacle negotiation ${ }^{1}$ system for mobile robots. Sensors suitable for obstacle negotiation are 2-D LADAR (i.e., a laser that scans in one plane) 3-D LADAR (i.e., a laser that scans and "nods," thus producing a range image of an area); Flash LADAR (i.e., a laser that has no moving parts but produces a range image of an area); stereo vision; and millimeter wave radar. Of these sensor modalities, 3-D laser scanners would be the best choice for building 3-D maps. However, 3-D scanners are inhibitively expensive for most mobile robot applications and many are not fast enough for map building in real time on a fast-moving vehicle, due to the relatively slow vertical scan. Flash LADAR, is fast, small, and less expensive, but our experience with early models has been disappointing with regard to resolution and errors. On the other end of the cost spectrum is stereo vision, which is the least expensive range sensing modality. Being a passive senor, it has very small power dissipation. However, the computation of stereo disparity relies on successfully matching a pixel in one camera to that in another camera. This operating principle limits its use to feature-rich environments. Other problems of stereo vision include sensitive to illumination and low range resolution, especially for distant objects.

A cost-effective alternative for 3-D mapping is to mount a 2-D laser scanner that is aimed forward and downward on the front end of a mobile robot. During motion the fanning beam of the scanner sweeps over the terrain in front of the robot, effectively creating a 3-D map. This approach is similar to our earlier developed Histogramic In-Motion Mapping algorithm [1], and a 3-D map with sufficient resolution for obstacle negotiation can be produced this way [9].

2-D laser scanners are widely used within the mobile robotics community and have been applied to object tracking [2], obstacle avoidance [3,4,5], feature extraction [6,7,8], map building [9,10] and self-localization $[11,12]$. Among the commercially available 2-D laser scanners, the LMS 200 made by the German company Sick [13] was the de-facto

\footnotetext{
${ }^{1}$ Obstacle negotiation is the capability of a mobile robot to decide whether an obstacle should be traversed or circumnavigated, and to execute the appropriate action. Obstacle negotiation is typically required in outdoor travel over rugged terrain.
} 
standard in the robotics community until the mid-2000s. However, in the early 2000s, the Japanese company Hokuyo [14] emerged on the scene with a series of LRFs. Among them are small-sized 2-D LRFs that appear to be more suitable for small robots than the Sick LMS 200.

The scientific literature offers several studies on the performance characteristics of LRFs. In 2002, two of the authors of the present paper, characterized the SICK LMS-200 laser rangefinder (LRF) [15]. In that paper, we measured the effect of the data transfer rate, drift, target surface properties, and incidence angles, and we developed a range measurement model specialized for mobile robot navigation.

Alwan et al. [16] characterized both the SICK LMS-200 and Hokuyo PBS-03JN and compared the result. Most of their work followed our procedure presented in [15]. Kawata et al. [17] characterized the Hokuyo URG-04LX. They focused on the reflectance of objects to adjust the sensor sensitivity. Pascoal et al. [18] characterized the SICK LMS-200 and the Hokuyo URG-04LX. They measured the effect of drift, surface properties, incidence angle, and accuracy of range measurements. In addition, they tested the sensors' ability to function in smoke-filled environments. However, the investigation on the measurement accuracy and the effect of incidence angles was limited to a white paper target. Lee, et al. [19] characterized the same two LRFs by measuring the effects of drift, target materials, and colors. They also measured the laser beam spot size and the effect of the targets' shape.

The unique contribution of our characterization study of the Hokuyo URG-04LX in this present paper is two-fold: (1) We used strictly the same experimental procedures and test apparatus for the Hokuyo LRF as we did in 2002 for the Sick LRF, and (2) we created a range measurement model that improves the accuracy of measured distances.

In the present paper, we investigate the Hokuyo URG-04LX LRF, shown in Figure 1. The Hokuyo LRF was developed by Hirohiko Kawata et al. [14] and has been commercially available since 2005. Table I compares some of the key characteristics of the Sick and Hokuyo LRFs.

This paper is organized as follows: In Section 2 we present a short functional overview and some technical specifications for the URG-04LX. In Section 3 we describe the experimental setup for our characteristization study. Section 4 investigates various characteristics of the Hokuyo LRF and in Section 5 we present a range measurement model derived from the experimental characterization results.

\section{THE HOKUYO URG-04LX LRF}

The Hokuyo URG-04LX is an LRF categorized as an Amplitude Modulated Continuous Wave (AMCW) sensor. As depicted in Figure 2, the laser emits an infrared beam and a rotating mirror changes the beam's direction. Then the laser hits the surface of an object and is reflected. The direction of reflected light is changed again by a rotating mirror, and captured by the photo diode. The phases of the emitted and received light are compared and the distance between the sensor and the object is calculated. A rotating mirror sweeps the laser beam horizontally over a range of $240^{\circ}$, with an angular resolution of $0.36^{\circ}$. As the mirror rotates at about $600 \mathrm{rpm}$, the scan rate is about $100 \mathrm{msec}$. The data transfer rate can be configured at 19.2, 57.6, 115.2, 250,500, or $750 \mathrm{Kbps}$ when it connected to an RS-232C port, or it can be set at

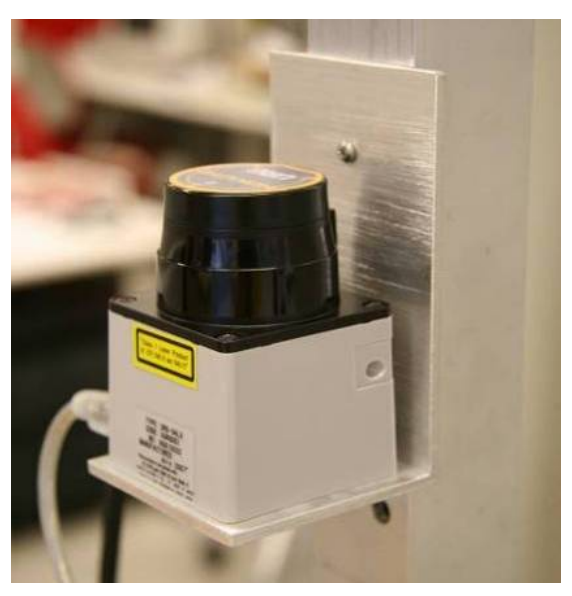

Figure 1: The Hokuyo URG-04LX Laser Rangefinder
Table I: Comparison of key specifications for the Sick and Hokuyo LRFs.

\begin{tabular}{|c|c|c|c|}
\hline & Units & $\begin{array}{c}\text { Sick } \\
\text { LMS-200 }\end{array}$ & $\begin{array}{c}\text { Hokuyo } \\
\text { URG-04LX }\end{array}$ \\
\hline Max range & $\mathrm{m}$ & 80 & 4.0 \\
\hline Resolution & $\mathrm{mm}$ & 10 & 1 \\
\hline Scan angle & deg & 180 & 240 \\
\hline Angular resolution & deg & $1 *$ & 0.36 \\
\hline Scan rate & scans/ sec & 75 & 10 \\
\hline PC interface & & RS-232/RS-422 & USB/RS-232C \\
\hline Data transfer rate & $\mathrm{KB} / \mathrm{sec}$ & $9.6-500$ & $\begin{array}{c}12,000(\mathrm{USB}) \\
19.2-750(\mathrm{RS}-232 \mathrm{C})\end{array}$ \\
\hline External Dimension & $\mathrm{mm}$ & $185 \times 156 \times 210$ & $50 \times 50 \times 70$ \\
\hline Weight & $\mathrm{kg}$ & 4.5 & 0.16 \\
\hline Power Consumption & $\mathrm{W}$ & 20 & 4.0 (maximum) \\
\hline
\end{tabular}

*Note: In interlaced scan mode, the sensor's angular resolution is $0.5^{\circ}$ or $0.25^{\circ}$ 
9 Mbps when connected via USB. The USB connection is thus much preferable and it was used for our tests. The LRF has a quoted range of between 20 and 4,095 $\mathrm{mm}$. The quoted measurement error is $\pm 10 \mathrm{~mm}$ for distances of less than $1 \mathrm{~m}$. For greater distances, the error is quoted as $\pm 2 \%$, assuming a target of a patch of white paper of size $70 \times 70 \mathrm{~mm}$. The measured range is discredited to 12-bits. If the sensor detects an error, an error code is sent with the same 12-bit range measurement. For example, the sensor emits error messages if the distance exceeds the limit, or if the received light is too strong or weak.

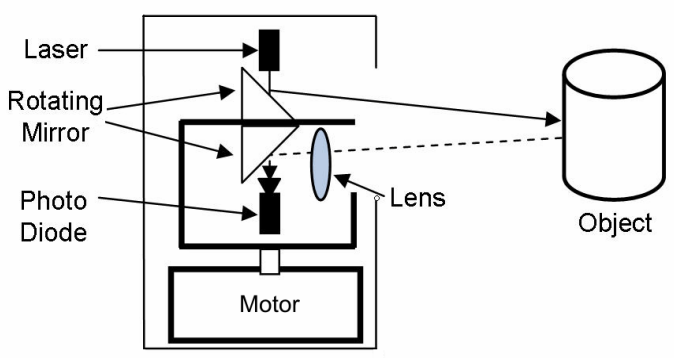

Figure 2: Operation principle of the Hokuyo LRF.

\section{EXPERIMENTAL SETUP}

In this section we present a procedure for calibrating the Hokuyo -LRF. The experimental setup for the calibration task is shown in Figure 3. The setup consisted of a linear motion table driven by a stepping motor, a target mounted on the sled of the linear motion table, a mounting pole for the LRF, and a computer that controls both the motion table and the data sampling from the LRF. Under computer control, the distance between the LRF and the target can be adjusted quickly and precisely. This allows for much of the time-consuming data collection to be done automatically. The operating system of the computer was Windows XP SP3, and the programs used for the experiments were created using the Visual $\mathrm{C}++2005$ Express Edition.

Due to mechanical tolerances in the mounting of the sensor, there is no guarantee that the nominal center beam (Beam \#384) of the sensor is actually parallel to the linear

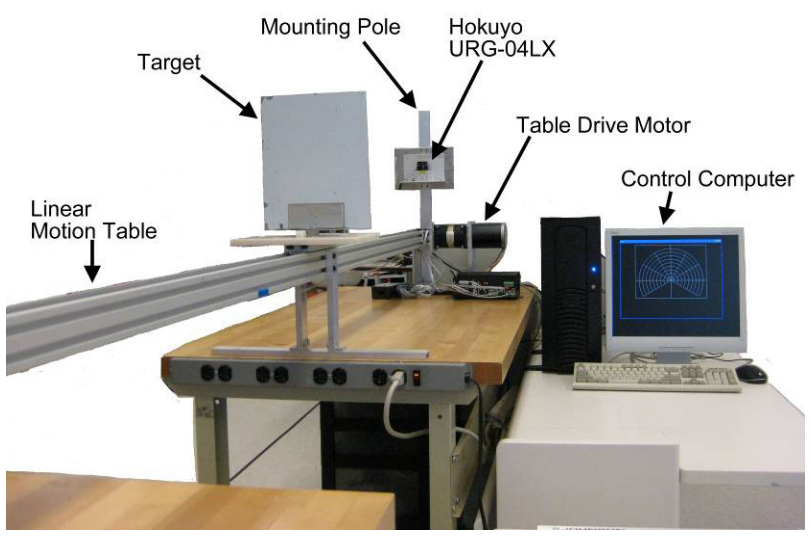

Figure 3: The experimental setup with a 4-meter long, computer-controller linear motion table. motion table. This misalignment must be corrected in order to obtain accurate range measurements. To correct the misalignment caused by mounting variations, we adjusted the orientation (pitch and yaw) of the LRF according to the following procedure before each experiment.

In order to establish vertical alignment, we set the target at a distance of $268 \mathrm{~mm}$ from the LRF. We sampled 10,000 scans and averaged the 10,000 range readings of Beam 384. This average was labeled " $d_{m l}$." The target was then moved $3,700 \mathrm{~mm}$ from its initial position and another 10,000 scans were taken. This time the average of readings for Beam 384 was labeled " $d_{m 2}$." Under conditions of perfect vertical alignment the difference $\Delta_{m}=d_{m 2}-d_{m 1}$ should equal 3,700 mm. If that was not the case, we inserted shim plates between the mounting pole and the LRF to change its pitch angle and the procedure was repeated, until $\Delta_{m}$ was closest to $3,700 \mathrm{~mm}$.

To establish horizontal alignment, we simply defined the beam that produced the shortest range to the target as the new, actual center beam. This was Beam \#386 in all our experiments and we subsequently used readings from this Beam to measure the distance to all targets.

\section{CHARACTERISTICS OF THE HOKUYO LRF}

In order to characterize the Hokuyo URG-04LX, we measured the data transfer rate as well as the effect of drift, surface properties, and incidence angles. All of these characteristics are relevant for mobile robotics applications. In our characterization experiments we used the same parameters as those used in Ye and Borenstein [18].

\subsection{Scan rate and data transfer rate}

The Hokuyo LRF can be connected to a PC through either RS-232 or USB. The data transfer protocol offered by the vendor is SCIP ver.1.0. Data for each complete scan is packaged as one data packet and each packet comprises 1,556 bytes ( 9 bytes for a command, 9 bytes for header, and 1,538 bytes for data). For the quoted scan rate of $10 \mathrm{~Hz}$, the 
maximal data throughput is $10 \times 1556=15.5 \mathrm{KBps}$. This is well below the maximum speed of both the RS-232 and the USB interface provided on the LRF. In all of our experiments we used the USB interface.

We wrote a simple C-program that performed 1,000 scans, while recording the time it took to complete each scan. The average scan rate, as computed from the results of this simple test, was $0.109 \mathrm{sec}$ or $9.17 \mathrm{~Hz}$, just under the specified scan rate of $10 \mathrm{~Hz}$.

\subsection{Warm-up Time}

In this experiment we measured the warm-up time for the LRF. The target was placed at a distance of approximately $2,000 \mathrm{~mm}$ from the LRF. Since the reference point for range measurements is not defined by the manufacturer, we considered the distance between the geometric center of the LRF and the object as the true range We scanned 600,000 times and calculated the average of each 1,000 measured ranges. Figure 4, shows the variations in the measured range. As is apparent, the range measurements stabilized after about 90 minutes. However, even during the first 90 minutes the range varied only by $6 \mathrm{~mm}$ or $0.3 \%$ of the distance measured. For most mobile robot applications, a range error of $0.3 \%$ is insignificant. Therefore, at room temperature, the Hokuyo LRF can be used without any warm-up. For the experiments described in this paper, however, we allowed 90 minutes of warm-up time prior to recording data. We did not test warmup time for ambient temperatures other than room temperature.

\subsection{Effect of target surface properties}

In order to characterize the Hokuyo URG-04LX LRF with regard to the different reflectance properties of different targets, we ran tests with 13 different targets organized in three groups. The target surface categories were:

Shiny colors: We used a cardboard with gold-colored reflective surface ${ }^{2}$, a cardboard with silver-colored reflective surface, and a sheet of polished aluminum ${ }^{3}$.

Matted colors: We used five cardboards with colored surfaces: blue, purple, red green, and yellow ${ }^{4}$.

Gray levels: We used standard white copier paper that was entirely covered with laser printer-produced gray-level output: RGB=255, RGB=191, RGB=127, $\mathrm{RGB}=63, \mathrm{RGB}=0$.

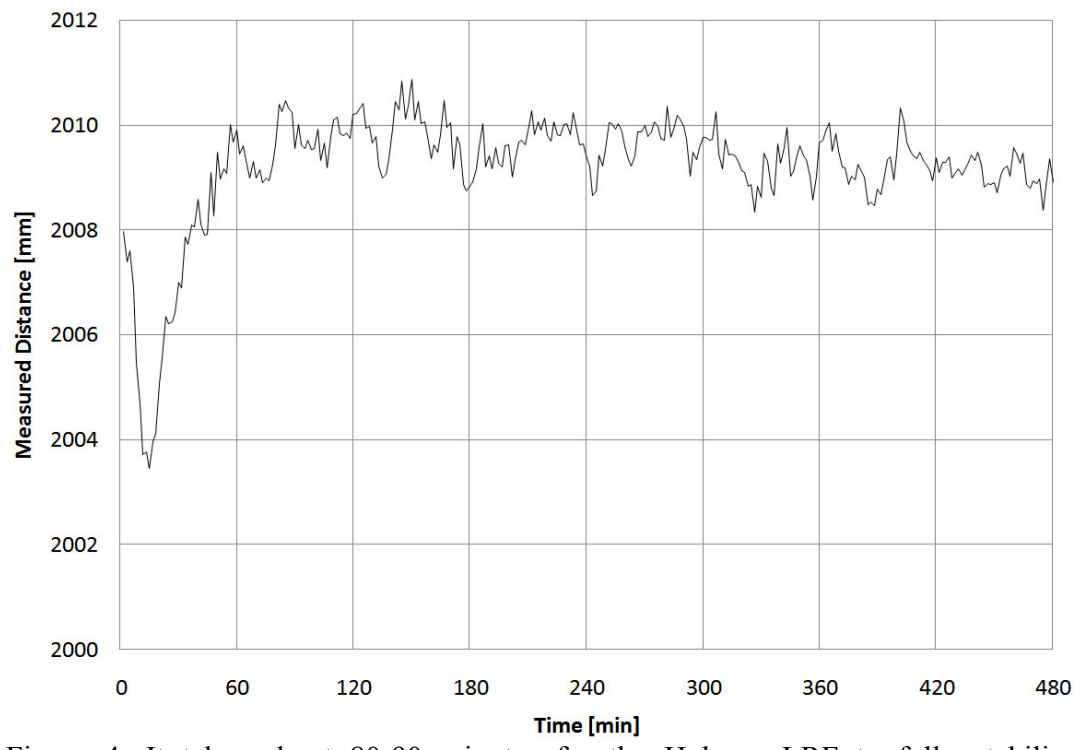

Figure 4: It takes about 80-90 minutes for the Hokuyo LRF to fully stabilize. However, variations during that warm up time are small. In our experiment variations were about $6 \mathrm{~mm}$ or $0.3 \%$ of the actual range of $2,000 \mathrm{~mm}$.

The targets were initially placed $268 \mathrm{~mm}$ in front of the LRF. During the experiment the motion table moved the targets further away, in 500-millimeter increments, up to a distance of $3,768 \mathrm{~mm}$. At each incremental position 10,000 samples were taken and the distribution of the measured range values was evaluated.

\footnotetext{
${ }^{2}$ Product name: Gold and Silver Foil with Cardstock backing. Vendor: www.michaels.com

${ }^{3}$ Product name: Polished Aluminum Plate (Material: \#5052, Finish: \#8), Part No. 8202K11. Vendor: www.mcmaster.com

${ }^{4}$ Product name: Cardstock Paper, "Purple”, "Red”, "Blue”, "Yellow”, “Green.” Vendor: DMD Inc.
} 
The measured distances for "shiny colors" have approximately normal distributions. However, the measured ranges for the gold target at $1,268 \mathrm{~mm}$, as well as those of the aluminum target at $1,768 \mathrm{~mm}$ and 2,768 $\mathrm{mm}$ are unreliable since the sensor issued a number of error codes along with these ranges. We found several notches on the peaks whose widths are $1 \mathrm{~mm}$. Since the notches occur at particular distances, such as 1,735 and $1,787 \mathrm{~mm}$, this phenomenon might be caused by a characteristic of the sensor. The shiny targets have relatively lower peaks and wider spreads than the "matted colors" and "gray levels" targets, and no significant difference in the width of the spreads and the height of peaks is observed among the shiny targets. The difference between the average of measured distance and true distance is relatively large for shiny targets, and standard deviations tend to be large. The maximum standard deviations for the full range $(268-3,768 \mathrm{~mm})$ are $\sigma_{\max }=$ 13.7, $\sigma_{\max }=7.9$, and $\sigma_{\max }=4.5$ for the gold, silver, and aluminum targets respectively. From the result, we conclude that the surface reflectivity affects range measurement. We believe that the sensor might not be tuned for the targets that have higher reflectivity.

The measured distances for "matted colors" targets and "gray level" targets have approximately normal distributions (Figure 5b and c). In contrast to the shiny targets, the measured distances have only one peak. However, outages similar to those of the shiny targets were observed at particular distances, of 778, 1273, 1761, and $1787 \mathrm{~mm}$ for both matted color and gray level targets. The gray level targets had an additional outage at $2,272 \mathrm{~mm}$.

The matted colors and gray level targets have relatively higher peaks and narrower spreads than the shiny targets, and no significant difference in the width of spread and height of peak is observed among the matted colors targets. The difference between the average of measured distance and true distance is relatively small, and the standard deviations are very small. The maximum standard deviation for all of the matted color and gray level targets is less than $4.5 \mathrm{~mm}$. Additionally, we believe that the measured range of the green target at $3,768 \mathrm{~mm}$ was unreliable since the sensor gave a number of error codes. This finding may not be a significant problem since the distance is close to the maximum range $(4,095 \mathrm{~mm})$. Thus, we conclude that surface color or gray level do not affect range measurement significantly.

In summary, the target surface reflectivity significantly influences both the mean value and the distribution of the measured distances. But the surface color and gray levels do not significantly influence the mean value or the distribution.

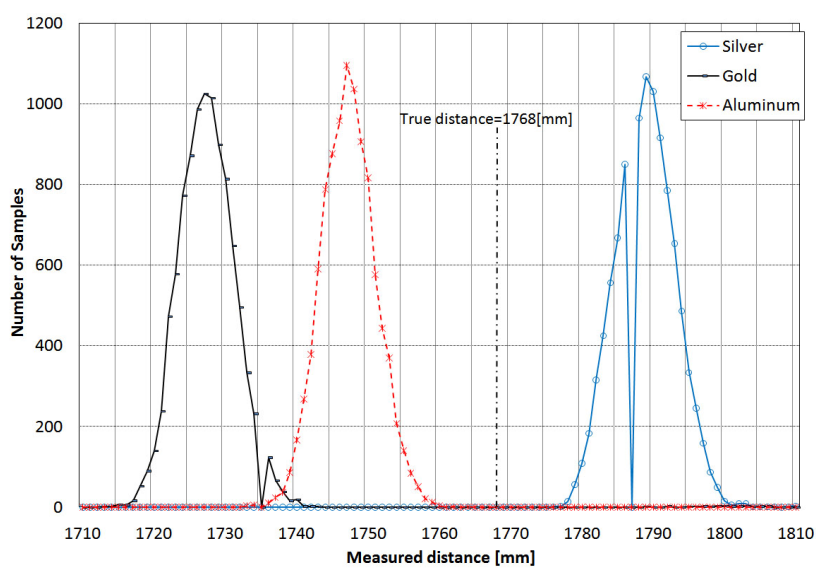

(a)

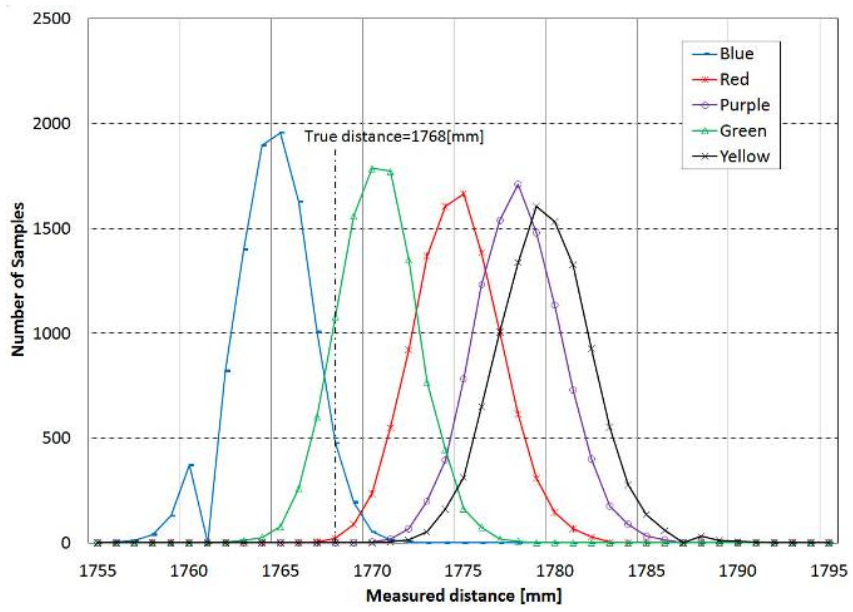

(b)

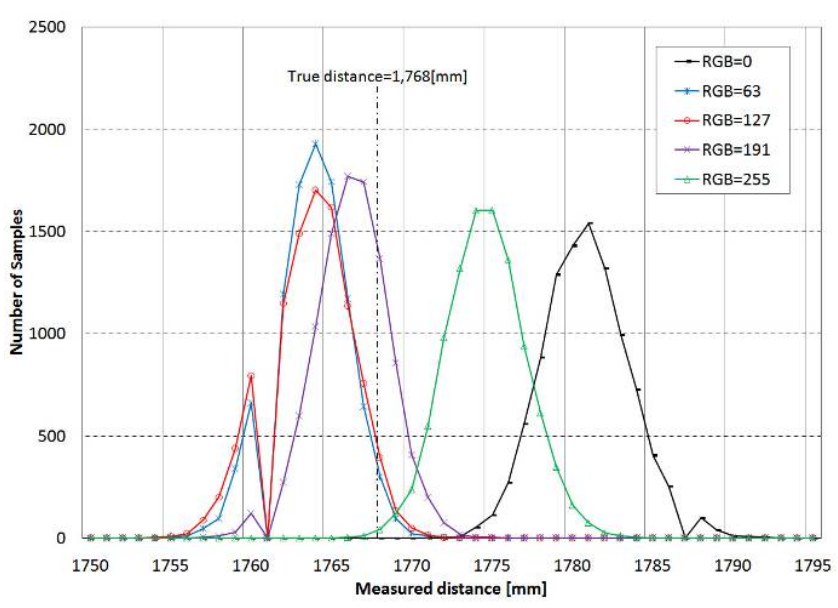

(c)

Figure 5: Distribution of measured distance. Shown are the distributions for the true distance of $1,768 \mathrm{~mm}$. (a) Shiny targets (aluminum is represented by a dotted line because it produced error codes for more than $1 \%$ of the readings), (b) matted colors targets, (c) gray levels targets 


\subsection{Effect of incidence angle}

In this section we examine the effect that different incident angles have on the range measurement accuracy. In the preceding section this angle was constant at $0^{\circ}$. One potential problem when conducting experiments with an incident angle other than $0^{\circ}$ is that the true distance between the scanner and the target is now harder to determine. This problem is illustrated in Figure 6: if both the scanner and the rotation axis of the target were located on the longitudinal center line of the linear table ( $\mathrm{a}$ and $\mathrm{b}$, respectively), then the true distance between the scanner and the target would always be $\overline{a b}$ regardless of the target's orientation. However, due to measurement errors during installation, we must assume that the scanner and the rotation axis of the target deviated by small distances ( $q$ and $p$, respectively, in Figure 6) from the linear motion table's longitudinal centerline. This introduces an additional error $\pm(p+q) \tan \theta$. Taking into account this error, the true distance is

$D=\overline{a b} \pm(p+q) \tan \theta$

where

$D$ - True distance between LRF and target

$\theta$ - Horizontal angular offset between the normal to the target surface and the center line of the linear motion table).

Since this error is not caused by an optical effect of the incidence angle or by the scanner it must be eliminated before measuring scanner errors. One way to eliminate this error is by computing the average of the pair of range values measured when the target is rotated horizontally by angles of $\theta$ and $-\theta$.

For this experiment, the gray target with $\mathrm{RGB}=127$ was positioned $2,000 \mathrm{~mm}$ in front of the scanner and 9 different orientation angles were set up using a protractor. These angles were: $0^{\circ}, \pm 10^{\circ}, \pm 20^{\circ}, \pm 30^{\circ}$, and $\pm 40^{\circ}$. We were not able to get reliable range measurements for $\theta \geq 40^{\circ}$. For each angle, 10,000 samples were taken. Then, the data pairs (e.g., data sampled at the positive and negative orientation) were averaged and the means of the measured range values were computed for $\pm 10^{\circ}, \pm 20^{\circ}, \pm 30^{\circ}$, and $\pm 40^{\circ}$, respectively. Figure 7 depicts the distributions of the measured ranges for the target at different orientations.

The shapes of the curves for $\pm 10^{\circ}, \pm 20^{\circ}$, and $\pm 30^{\circ}$ look almost symmetric, and the corresponding peaks are close to each other. The measured range is quite close to the true range, with an error of about $14 \mathrm{~mm}$ or $0.7 \%$.

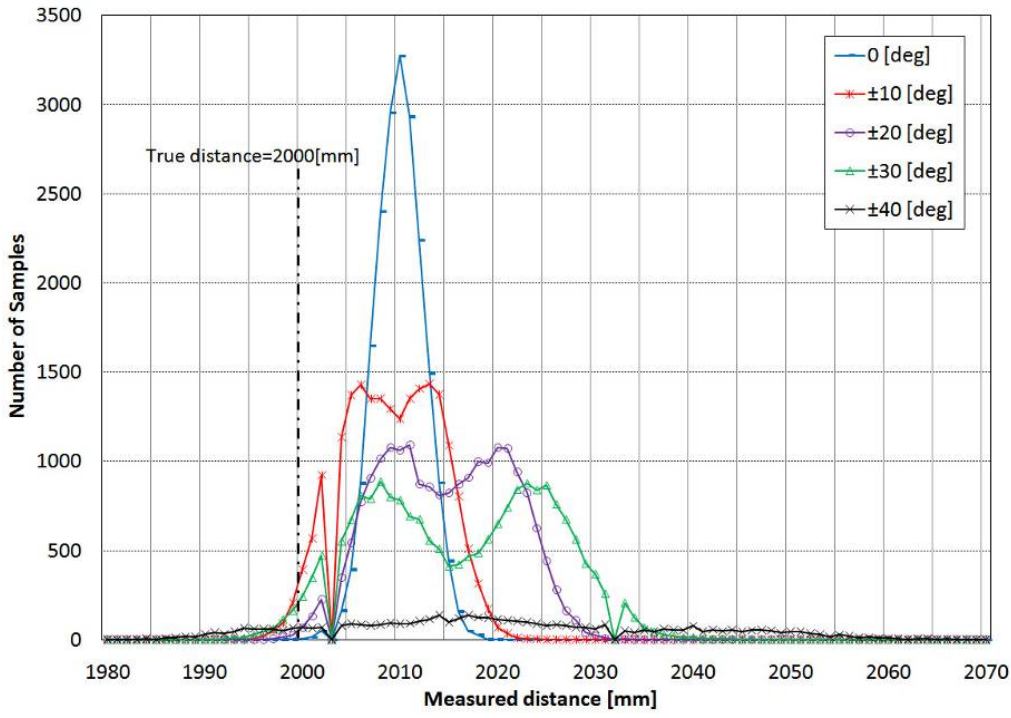

Figure 7: Distribution of ranges at various horizontal orientations for the gray target with RGB-127.

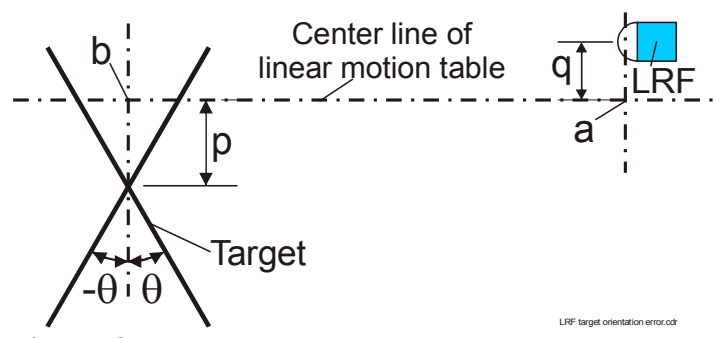

Figure 6:

Additional error introduced by object orientation.

We therefore conclude that an incidence angle between $-20^{\circ}$ and $+20^{\circ}$ does not cause significant errors in the range measurements. By contrast, at incidence angles of $\pm 30^{\circ}$ and beyond, the LRF issues error codes and the measured ranges becomes unreliable. In conclusion, the incidence angle has less influence than the color and gray levels of targets if the incidence angle is within $\pm 30^{\circ}$.

Other targets, namely RGB=191, RGB $=255$, Yellow, Purple, Blue, and Red all performed better than $\mathrm{RGB}=127$. For those targets the significant errors occurred only if the LRF couldn't detect it at incident angles beyond than $\pm 50^{\circ}$. On the other hand, the $\mathrm{RGB}=63$ target performed worse than $\mathrm{RGB}=127$. The significant errors occurred if the incident angles are beyond $\pm 10^{\circ}$. For the $\mathrm{RGB}=0$ as well as the gold and silver target, the LRF couldn't detect the target if the incident angles was greater than $\pm 0^{\circ}$. 


\subsection{The mixed pixels problem}

When a laser spot is located at the very edge of an object, the measured range is that of a combination of the foreground object and the background object, i.e., the range falls in between the distances to the foreground and background objects. This condition is called "mixed pixels" [13]. To investigate this condition, we positioned a target at about $1,100 \mathrm{~mm}$, and a uniform background at about $2,000 \mathrm{~mm}$ in front of the scanner. At this distance 34 beams (from beam $\# 372$ to \#405) hit the target, while all other beams hit the background.

Figure 8 shows a plot of the mean from 250 scans in Cartesian coordinates. One mixed pixel, labeled ' $a$ ', was found at 1,829 $\mathrm{mm}$. One should note that the mixed pixels phenomenon exists even in a single scan, that is, it is not the result of averaging values of multiple scans.

Since the mixed pixels condition occurs when the laser spot is located at an area where the reflectivity of the surface changes sharply, the phenomenon is not rare. In our experiments, mixed pixels occurred at a variety of distances between the LRF and the target.

\section{Calibration And Simulation Models}

Based on our empirical data we built a mathematical model of the scanner errors. This model can be used as a calibration function to reduce measurement errors.

\section{$5.1 \quad$ The calibration model}

To calibrate the measured range values, the targets were placed at a position from $y_{1}=268 \mathrm{~mm}$ to $y_{186}=3,768 \mathrm{~mm}$ in increments of $20 \mathrm{~mm}$. At each position $y_{i}, 4,000$ samples, $z_{j i}$ for $j=1, \ldots, 4,000$, were taken and the mean, denoted as $\mu_{i}$, was calculated. The 13 targets introduced in Section 4 were used to collect the data. We found that the mean had an approximate linear relationship with the true distance for each target. Therefore, the true distance may be estimated by a linear function as follows:

$$
\hat{y}=k \mu+b
$$

where

$\hat{y}$ - estimate of the true distance $y$,

$\mu \quad$ - mean of the measured ranges

$k, b$ constants for minimizing the square error, which is defined as

$$
\sum_{i=1}^{n}\left(y_{i}-\hat{y}_{i}\right)^{2}=\sum_{i=1}^{n}\left(y_{i}-k \mu_{i}-b\right)^{2}
$$

Therefore, we have

$$
k=\frac{\sum_{i=1}^{n}\left(y_{i}-\bar{y}\right)\left(\mu_{i}-\bar{\mu}\right)}{\sum_{i=1}^{n}\left(\mu_{i}-\bar{\mu}\right)}
$$


and

$$
b=\bar{y}-k \bar{\mu}
$$

where $\bar{y}$ and $\bar{\mu}$ are the mean values of $y_{i}$ and $\mu_{i}$, respectively.

Ideally, the calibration should account for the orientation and the surface properties of the targets. However, surface properties are not usually known in most mobile robot obstacle negotiation applications. Therefore, for our application we use only the data acquired for a medium gray target $(\mathrm{RGB}=127)$ at $0^{\circ}$ incidence angle to calibrate the measured ranges. With this data $k=1.0102$ and $b=-7.0$.

Eq. (2) can be used as a calibration function that corrects range measurements for empirically determined errors of the scanner. We use this function to calibrate the measured ranges of the 13 targets and the result is depicted in Figure 9. Figure $9 \mathrm{a}$ shows the range error $\hat{y}-y$ vs. the true distance $y$ and Figure $9 \mathrm{~b}$ depicts the standard deviations. The range errors for the 13 different targets have an upper bound of $61 \mathrm{~mm}$ for "Shiny Colors," $32 \mathrm{~mm}$ for "Matted Colors," and $30 \mathrm{~mm}$ for "Gray Levels." The lower bound is $-137 \mathrm{~mm}$ for "Shiny Colors," $-22 \mathrm{~mm}$ for "Matted Colors," and $26 \mathrm{~mm}$ for "Gray Levels." The standard deviations are bounded by $(2.6,121)$ for "Shiny Colors." $(1.8,5.2)$ for "Matted Colors." and $(1.8,5.4)$ for "Gray Levels. These values are larger than the specifications provided by the manufacturer.

\subsection{The range measurement model}

The range measurement model is a simulation model, which estimates the measured range using the true distance. It may be used to simulate the scanner function in simulations.

Since the mean of the measured range has an approximately linear relationship with the true distance, it may be approximated by a linear function:

$\hat{\mu}=n y+c$

where $\hat{\mu}$ is the estimate of $\mu$ and $y$ is the true distance; while $n$ and $c$ are constants. Following the same method as in the preceding section, we find $n$ and $c$ from

$n=\frac{\sum_{i=1}^{n}\left(y_{i}-\bar{y}\right)\left(\mu_{i}-\bar{\mu}\right)}{\sum_{i=1}^{n}\left(y_{i}-\bar{y}\right)}$

and

$c=\bar{\mu}-n \bar{y}$

Assuming the measurement error is Gaussian noise, the simulated measured range value of a single scan is

$z_{s}=\operatorname{Int}(n y+c+\xi)$

where $\xi$ is Gaussian noise with a normal distribution $N(0, \sigma)$. The rounding function $\operatorname{Int}()$ is applied to the measurement model, since the physical LRF produces integer range values.

We also used the data acquired by a medium gray target $(\mathrm{RGB}=127)$ at $0^{\circ}$ incidence angle to model the range measurements. With this data set, $n=0.9899$ and $c=7.0$. The value of $\sigma$ in Eq. (6) determines how closely the simulation model matches the physical scanner. We used the calibrated range measurements of the target at distances from $2,048 \mathrm{~mm}$ to $2,648 \mathrm{~mm}$. In this range, the minimum and maximum standard deviations are 2.2 and 3.3 , respectively. The process for determining the value of $\sigma$ is as follows:

1. The distribution of the calibrated range measurements - measured by the physical scanner and calibrated by Eq. (2) - for each distance from 2,048 $\mathrm{mm}$ to 2,648 $\mathrm{mm}$ was calculated.

2. The simulated range measurement was computed by Eq. (7) where $y$ assumes value from 2,048 $\mathrm{mm}$ to $2,648 \mathrm{~mm}$ in increments of $20 \mathrm{~mm}$ and $\sigma$ from 2.2 to 3.3 in increments of 0.1 . For each position and $\sigma$ value, 4,000 simulated range values were calculated and the distribution was computed. 
3. The mean square error between these two distributions was calculated for each $\sigma$ by

$$
E S=\frac{1}{31} \sum_{i=1}^{31} \sum_{j=-2}^{2}\left(N S_{i}(j)-N R_{i}(a)\right)^{2}
$$

where $N S_{i}$ and $N R_{i}$ are the number of samples for the simulated and real distributions, respectively. The parameter $i$ is the index of the position from $1,992 \mathrm{~mm}$ to 2592 while $j$ is the index of the five most significant numbers of samples. The mean square error achieved its minimum at $\sigma=2.9$. Therefore, $\sigma=2.9$ is used to model the Gaussian noise for Eq. (7).

After the values of $n, c$ and $\sigma$ were determined, the model of Eq. (7) was validated with other sets of range measurements. In all cases the mean square error computed by Eq. (8) was sufficiently small to validate our approximation assuming Gaussian noise.

\section{CONCLUSIONS}

In this paper, the Hokuyo URG-04LX LRF was characterized through extensive experimentation. Our experiments suggest that the output of the LRF takes up to 90 minutes after start-up to stabilize. However, the measured range to a target varied by no more than $0.3 \%$ of that range. For typical mobile robot applications this variation is insignificant. We also found that-similar to other LRFs - so-called mixed pixels may occur in range measurements. In obstacle avoidance applications mixed pixels are a concern because even a single false positive (i.e., indicating a point as occupied by an obstacle whereas in reality the point is not occupied) may "block" a path that is actually wide open.

Because of the mixed pixel problem, it is necessary to use statistical models to represent the environment. By using an appropriate statistical model, a lone mixed pixel will not block a path or distort a map significantly.

Other measurement errors are caused by target surface reflectance properties and the incidence angle of the laser beams. Maximal errors were approximately $140 \mathrm{~mm}$ for "Shiny Colors," $30 \mathrm{~mm}$ for "Matted Colors," and $32 \mathrm{~mm}$ for "Gray Levels." These errors are acceptable for most mobile robot applications. Errors resulting from incident angles other than normal ones were small (approximately $14 \mathrm{~mm}$ ) for most colored or dark targets if the incidence angle is within $\pm 30^{\circ}$. However, white and shiny surfaces can produce specular reflections for incident angles beyond $\pm 10^{\circ}$. This is a significant concern for obstacle avoidance applications. In our experience, the tendency of a sensor and sensor-tosurface constellation to produced specular reflections typically forces a reduction in the robot's operating speed. This is because objects that produce specular reflections from a large distance become "visible" only as the robot comes closer to the obstacle and beams with more favorable incident angles "see" the obstacle. It is this need to approach an obstacle closely to "see" it (often much closer than the maximal range of the sensor), combined with the Hokuyo's comparatively slow scan rate of $10 \mathrm{~Hz}$ that forces slower operating speeds for a robot using that LRF. 


\section{Acknowledgements}

This work was supported by the U.S. Department of Energy under Award No. DE FG52 2004NA25587.

\section{REFERENCES}

[1] Borenstein, J. and Koren, Y., "Histogramic in-motion Mapping for Mobile Robot Obstacle Avoidance," IEEE Trans. on Robotics and Automation, 7(4), 535-539 (1991).

[2] Kogut, G. et al., "Sensor Fusion for Intelligent Behavior on Small Unmanned Ground Vehicles," Proc. SPIE 6561, (2007).

[3] Chang, Y., Kuwabara, H and Yamamoto, Y., "Novel Application of a Laser Range Finder with Vision System for Wheeled Mobile Robot," Proc. of IEEE/ASME Int. Conf. on Advanced Intelligent Mechatronics, 280-285 (2008).

[4] Zeng, S. and Weng, J., "Online-learning and Attention-based Approach to Obstacle Avoidance Using a Range Finder," Journal of Intelligent Robot System 50, 219-239 (2007).

[5] Choi, Y., and Hong, J. and Park, K., "Obstacle Avoidance using Active Window and Flexible Vector Field with a Laser Range Finder," Int. Conf. on Control, Automation and Systems, 2123-2128 (2007).

[6] Borges, G. and Aldon, M., "Line Extraction in 2D Range Images for Mbile Robotics," Journal of Intelligent and Robotic Systems, 40, 267-297 (2004).

[7] V. Nguyen, et al., "A Comparison of Line Extraction Algorithms using 2D Laser Range Finder for Indoor Mobile Robotics," IEEE/RSJ Int. Conf. on Intelligent Robots and Systems, 1929- 1934 (2005).

[8] Premebida, C. and Nunes, U., "Segmentation and Geometric Primitives Extraction from 2D Laser Range Data for Mobile Robot Applications," Robotica 2005, Actas do Encontrol Cientifico, (2005).

[9] Ye, C. and Borenstein, J., "A New Terrain Mapping Method for Mobile Robots Obstacle Negotiation," Proc. SPIE, 52-62 (2003).

[10] Ueda, T. et al., "Mobile SOKUKI Sensor System: Accurate Range Data Mapping System with Sensor Motion," Int. Conf. on Autonomous Robots and Agents, 309-314 (2006).

[11] Sohn, H. and Kim, B., "A Robust Localization Algorithm for Mobile Robots with Laser Range Finders," Proc. IEEE Int. Conf. on Robotics and Automation, 3545-3550 (2005).

[12] Lingemann, K. et al., "High-speed Laser Localization for Mobile Robots," Robotics and Autonomous Systems, 51, 275-296 (2005).

[13] SICK Inc., “http://www.sick.com.” (2009)

[14] Hokuyo Automatic Co. Ltd, “http://www.hokuyo-aut.jp,” (2009)

[15] Ye, C. and Borenstein, J., "Characterization of a 2-D Laser Scanner for Mobile Robot Obstacle Negotiation," Proc. IEEE Int. Conf. on Robotics and Automation, 2512-2518 (2002).

[16] Alwan, M. et al., "Characterization of Infrared Range Finder PBS-03JN for 2-D Mapping," Proc. IEEE, Int. Conf. on Robotics and Automation, 3936-3941 (2005).

[17] Kawata, H. et al., "A Method for Estimation of Lightness of Objects with Intensity Data from SOKUIKI Sensor," Proc. IEEE INT. Conf. on Multisensor Fusion and Integration for Intelligent Systems, 661-664 (2008).

[18] Pascoal, J., Marques, L. and Almeida, A., “Assessment of Laser Range Finders in Risky Environments,” IEEE/RSJ Int. Conf. on Intelligent Robots and Systems, 3533-3538 (2008).

[19] Lee, K. and Ehsani, R., "Comparison of Two 2D Laser Scanners for Sensing Object Distances, Shapes, and Surface Patterns," Computers and Electronics in Agriculture, 60, 250-262 (2008). 